\title{
MRI-pathological correlate of brain lesions in a necropsy case of HTLV-I associated myelopathy
}

\author{
Akihiko Ogata, Kazuo Nagashima, Kunio Tashiro, Akira Miyakawa, Chikara Mikuni
}

\begin{abstract}
A postmortem case of HTLV-I associated myelopathy (HAM)/tropical spastic paraparesis (TSP) with a history of remission and exacerbation of neurological signs and symptoms, resembling those of multiple sclerosis is reported. MRI analysis revealed lesions in the periventricular white matter in addition to atrophy of the thoracic spinal cord, characteristic of HAM/TSP. The cerebral periventricular areas consisted of ill-defined paucity of myelin sheaths with astrocytic gliosis and hyaline thickening of blood vessels. The poorly demarcated white matter lesions found in both brain and spinal cord were different from plaques found in multiple sclerosis. It is suggested that, in some cases of HAM/TSP, inflammatory lesions that destroy myelin can involve not only the spinal cord but also the cerebral periventricular white matter.
\end{abstract}

(F Neurol Neurosurg Psychiatry 1993;56:194-196)

Previous reports on necropsy cases of HTLV-I associated myelopathy (HAM)/tropical spastic paraparesis (TSP) ${ }^{1.5}$ suggested that the cardinal neuropathological change in HAM/TSP was inflammatory destruction of the thoracic spinal cord. In some patients with HAM/TSP there was no cerebral abnormalities on MRI. ${ }^{6}$ But multiple sclerosis like lesions in the cerebral white matter on MRI in HAM cases have been reported. ${ }^{7-10}$ No one has provided a definitive explanation as to whether these cerebral white matter lesions are the same as, or different from, multiple sclerosis plaques. In this report on a case of HAM, we describe the clinical and pathological findings in the brain and spinal cord and compare these with the MRI findings. The involvement of the cerebrum in HAM is discussed.

\section{Case Report}

The patient was a 61 year old man from Sapporo on the Japanese island Hokkaido. He had not received any blood transfusions. When he was 25 years old, the patient began to experience many episodes of low back pain and gait disturbance, which occurred for a few weeks and then disappeared.

When he was 42 years old, the patient developed hand tremor, low back pain, gait disturbance, paresthesia of both legs, urinary disturbance, and vertigo. At that time, myelo- graphy and radiographs of the lumbar spine revealed no abnormalities. The symptoms soon regressed without treatment and he was able to walk by himself. The tentative diagnosis was multiple sclerosis. Thereafter, the patient required assistance in walking because of spastic paraparesis.

At the age of 56 years, he noticed swelling of the left submaxillary lymph node. A biopsy revealed malignant lymphoma of the diffuse large cell type of B-cell origin. This was confirmed by immunohistochemical staining with monoclonal antibody, L-26, which reacts with lymphoid cells of B-cell lineage. Prednisolone, cyclophosphamide, and vincristine were administered. During treatment with these drugs, the neurological symptoms regressed but the patient's condition became worse after the drugs were discontinued.

Neurological examination at the age of 60 years revealed spastic paraparesis, hyperreflexia of both legs with pathological reflex and ankle clonus, sensory disturbance below T-8, and a decreased vibration sense at the ankles, knees and hips. MRI showed T2-weighted, high signal intensity areas in the periventricular white matter of the cerebrum, especially in the white matter around the posterior horn of the lateral ventricle (fig 1). MRI also disclosed cerebral atrophy and the atrophy of the thoracic spinal cord. Serum and CSF antibodies to HTLV-I were positive at titres of $\times 2560$ and $\times 128$, respectively, by immunofluorescence assay. Prednisolone was administered for more than one year at a dose of $20 \mathrm{mg}$ per day. At the age of 61 years, the patient again underwent chemotherapy, as well as radiation therapy (40 Gy), for swelling of the right submaxillary lymph node. Despite this treatment, the patient died of recurrence of malignant lymphoma and bronchopneumonia.

\section{Pathological findings}

Necropsy was performed two hours after death. General postmortem findings were interstitial pneumonia of both lungs, multiple gastric ulcers, and malignant lymphoma, nodules of which were disseminated in the great curvature of the stomach, the left adrenal gland, the pancreatic body and the epicardium. The brain weighed $1350 \mathrm{~g}$ and was unremarkable externally, except for mild sulcal widening and moderate atherosclerosis of the vertebrobasilar artery. The lower thoracic spinal cord showed severe atrophy.

Pathological changes were found in the lower thoracic cord. The spinal cord lesions 


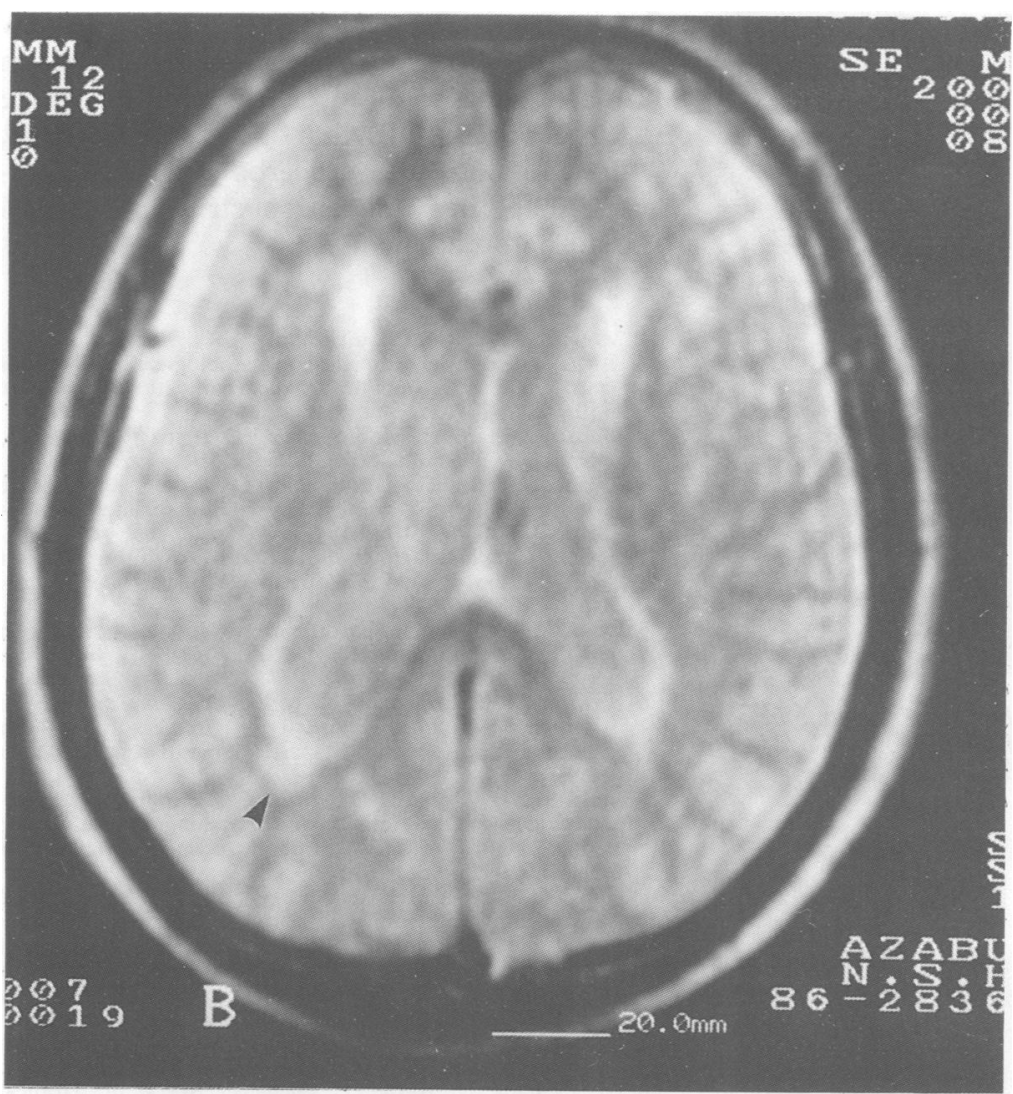

Figure 1 MRI scan showing T2-weighted, high signal intensity areas in the periventricular white matter, especially around the posterior horn of the lateral ventricle (arrow).

consisted of a loss of myelin sheaths and axons, principally involving the lateral columns and, to a lesser degree, the posterior columns, the posterior spinocerebellar tract and the anterior funiculus (fig 2). These lesions were ill defined and there was no demarcation of the demyelinated foci which is a characteristic feature of multiple sclerosis. There was hyaline thickening of blood vessels in the affected portion of the spinal cord, with astrocytosis. Perivascular

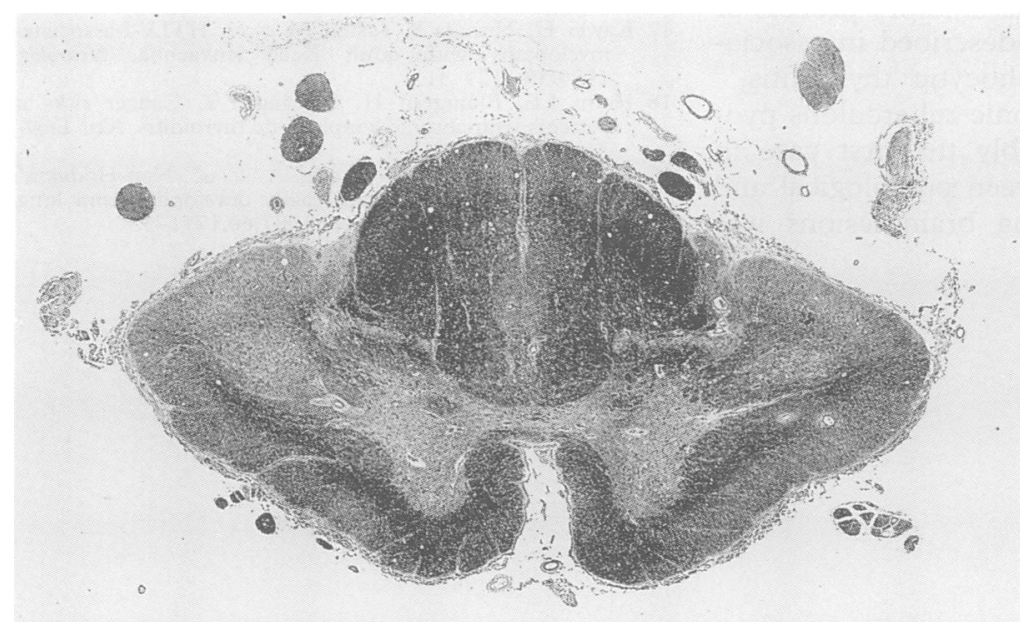

Figure 2 Affected thoracic spinal cord (T-6), revealing loss of myelin and axon, predominantly in the lateral columns and to a lesser degree in the posterior columns, and also in the posterior spinocerebellar tract and anterior funiculus. Klüver-Barrera stain. $\times 15$. and parenchymal inflammatory cell formation was not observed. There were few foamy macrophages and capillary proliferation was absent. Posterior and anterior nerve roots were well preserved. In the lumbar and cervical cords the lateral column was affected preferentially, but the severity of the loss of axons and myelin sheaths was much less than that in the thoracic cord.

In the periventricular white matter of the brain which corresponded to MRI findings, there was an ill defined lesion where the myelin sheaths decreased with astrocytic gliosis. The axons were also slightly decreased in number. Small vessels in this area showed hyaline thickening (fig 3). This vascular change was never seen in other areas of the brain. No inflammatory cell infiltration was found. These pathological features in the brain were essentially similar to those in the spinal cord. No intranuclear or intracytoplasmic inclusion bodies, multinucleated giant cells, or pathognomonic neuronal degeneration was found either in the brain or in the spinal cord.

\section{Discussion}

Gessain et $a l^{11}$ indicated that approximately $60 \%$ of TSP patients have antibodies to HTLV-I in their serum, and they suggested that HTLV-I infection might contribute to the pathogenesis. Vernant et al ${ }^{12}$ proposed HTLVI associated TSP as a new clinical and epidemiological entity from the study of 25 patients with the disease. Since Osame et al $^{13}$ reported similar cases in Japan, many cases with similar myelopathy and high titres of HTLV-I antibody in the serum and CSF have been reported in Japan. From the necropsy findings, ${ }^{1-5}$ HAM was regarded on pathological grounds as a disease different from multiple sclerosis.

Our case was compatible with HAM with regard to high titres of HTLV-I antibodies in both serum and CSF, spastic paraparesis and improvement by steroid therapy. Interestingly, in the early stage of his disease, the patient had exacerbations and remissions resembling the clinical course of multiple sclerosis.

The MRI findings in this case revealed areas of high signal intensity in the periventricular white matter, predominantly posterior to the lateral ventricles, with lesions similar to those found in multiple sclerosis. ${ }^{14}$ By MRI, Mattson et $a l^{7}$ detected mild and asymptomatic CNS lesions related to HTLV-I infection. Hara et $a l^{8}$ and Tashiro et $a l^{9}$ reported spotty areas in the white matter of the brain identified by MRI. These findings were not accurately distinguishable from those found in the plaques of multiple sclerosis. Moreover, HAM patients showed the same percentage of abnormalities in the periventricular areas as controls, due to ageing. ${ }^{15}$

We attempted to establish a clinico pathological correlation in this case by means of serial sectioning of the periventricular white matter lesions detected by MRI around the posterior horn of the lateral ventricles. Hyaline thickening of the small vessels, demyelination, slightly affected axons and poorly demarcated 


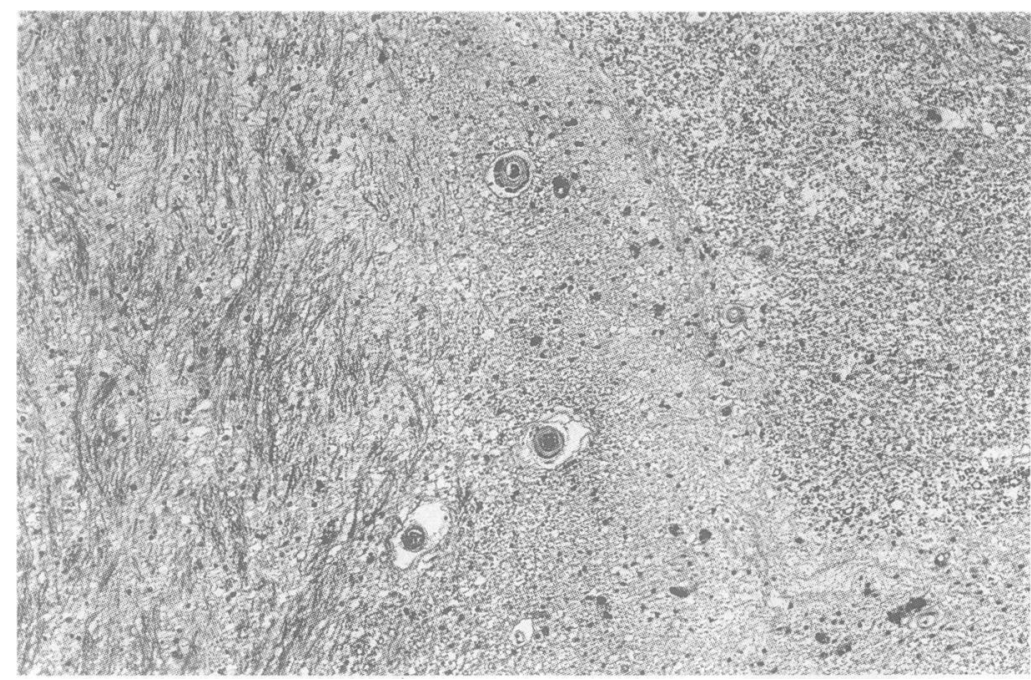

Figure 3 Ill-defined demyelination with gliosis is seen in the white matter around the posterior horn of the lateral ventricle. In the lesion, hyaline thickening of small blood vessels is observed compared with the control, but there is no inflammatory cell infiltration, Klüver-Barrera stain. $\times 100$. documented. These observations provide new information for research on brain lesions in HAM patients. Moreover, the clinical course in this case resembles that of multiple sclerosis, with remissions and exacerbations. This indicates that the clinical spectrum of HAM should be broadened.

1 Montgomery RD, Cruickshank EK, Robertson WB McMenemy WH. Clinical and pathological observation on Jamaican neuropathy. A report on 206 cases. Brain 1964;87:425-62.

2 Mani KS, Mani AJ, Montgomery RD. A spastic paraplegic syndrome in South India. $\mathcal{F}$ Neurol Sci 1969;9:179-99.

3 Akizuki S, Setoguchi M, Nakazato $O$ et al. An autopsy cas of human T-lymphotropic virus type I-associated myelopathy. Hum Pathol 1988;19:988-90.

4 Furuzono $\mathrm{H}$, Nakazato $\mathrm{O}$, Goto $\mathrm{K}$ et al. An autopsy case of HTLV-I-associated myelopathy (HAM). Clin Neurol (尹pn) 1989;29:349-54.

5 Iwasaki Y. Pathology of chronic myelopathy associated with HTLV-I infection(HAM/TSP). $\boldsymbol{f}$ Neurol $S_{c}$ 1990;96:103-23.

6 Newton M, Cruickshank K, Miller D et al. Antibody to human T-lymphotropic virus type 1 in West-Indian-born UK residents with spastic paraparesis. Lancet UK residents

7 Mattson DH, McFarlin DE, Mora C, Zaninovic V. Centralnervous-system lesions detected by magnetic resonance imaging in an HTLV-I antibody positive symptomles individual. Lancet 1987;ii:49.

8 Hara Y, Takahashi M, Yoshikawa H et al. HTLV-I associated myelopathy with multiple spotty areas in cerebral white matter and brain stem by MRI. Clin Neurol (7pn) 1988;28:92-6.

astrocytic gliosis were observed in these lesions. These pathological features of the lesions in the periventricular white matter resembled those of the spinal cord lesions, which suggested HAM. The lack of perivascular inflammatory cell infiltration could be attributed to the prolonged steroid therapy and to the long clinical course, which extended over 19 years. The patient's definitive age at the time of onset of the disease was estimated at 42 years. There was no history of blood transfusion which might have contributed to the slowly progressive long clinical course.

Usuku et $a l^{16}$ reported on the different immunogenetic backgrounds responsible for susceptibility to HAM and adult T-cell leukaemia/lymphoma, but a case of a patient with both has been reported only once recently. ${ }^{17}$ B-cell lymphoma in our patient could be related to the chronic inflammatory process of HAM/TSP, as has been described in association with chronic lymphocytic thyroiditis, ${ }^{18}$ and a long-standing chronic tuberculous pyothorax. ${ }^{19}$ Ours is probably the first case in which a correlation between pathological and MRI findings concerning brain lesions was 